\section{Mascotas felinas, medicina y arte}

\author{
FELIPE CABELLO C. ${ }^{1}$
}

\section{Pet cats, medicine and art}

The author in this short text remembers the passing of a beloved pet cat. Cats are beautiful animals and wonderful companions. They evolved together with humans in Africa, subsequently spread over the five continents with them, and became the subject of many human cultural activities, including art, poetry and religion. Abandoned and roaming free, cats readily return to the wild, potentially acquiring many zoonotic infections. Surprisingly, feline company is increasingly used for therapy of mental and other illnesses such as cardiovascular disorders. Responsible ownership and good veterinary care of these marvelous animals under the One Health paradigm are essential to their well-being as well as to that of humans. They are an ethical and small price to pay for the affectively rewarding relationships humans have with these endearing and evocative animals.

(Rev Med Chile 2019; 147: 91-95)

Key words: Cats; Cat diseases; Medicine in the arts; Pets.
${ }^{1}$ Department of Microbiology and Immunology. Estados Unidos de Norteamérica.

Correspondencia a:

Felipe Cabello C

New York Medical College,

Valhalla NY 10530

Estados Unidos de Norteamérica. cabello@nymc.edu on mi experiencia en microbiología y en medicina, podría escribir en este breve ensayo para recordar a una gatita regalona en un contexto médico, acerca de las zoonosis bacterianas (alrededor de 17), virales (a lo menos 2 ), fúngicas (3-4) y parasitarias (5), que pueden ser trasmitidas de las mascotas felinas a los humanos $^{1-4}$. Sin embargo, tal ejercicio académico fracasaría en representar el amplio cariño y magia que la gata regalona fue capaz de inspirar y también ignoraría que todas estas zoonosis son prevenibles empleando el productivo concepto ecológico de Una Salud ${ }^{5}$. El ejercicio también limitaría la descripción de las relaciones de los humanos con los felinos a solo un aspecto de ella, tal vez el menos importante para el objetivo de este ensayo. Desde su domesticación, en el Oriente Medio, miles de años atrás, los gatos además de ser animales domésticos de compañía, útiles en el combate contra los roedores y sus depredaciones, también han sido y son para los humanos animales simbólicos y de gran significado emocional ${ }^{6-8}$. Esto porque ellos siempre han sido sujetos de admiración por su original gracia y elegancia y, además, porque permanentemente han sido protagonistas de nuestra imaginación en la literatura, en la cinematografía, en el teatro, en la música e igualmente en las religiones y en las creencias populares de diversas sociedades ${ }^{6-8}$. La evolución y la domesticación de estos animales han estado íntimamente ligadas a la evolución biológica y cultural de los humanos y a su historia $^{6-8}$. Por esto es, que en los últimos años, con las nuevas técnicas de filogenia y genómica arqueológica ha habido un renovado interés en develar estas interacciones recíprocas y en identificar orígenes geográficos y culturales comunes en el desierto africano y el Oriente Medio y su migración coetánea hacia el resto del mundo desde estos lugares ${ }^{6,7}$. Aparentemente, los ancestros de los gatos actuales son el resultado de la domesticación de la especie silvestre del desierto africano Felix silvestris lybica, que a través de los siglos se hibridizó genéticamente y en numerosas oportunidades, con otras especies de felinos silvestres del Viejo Continente $e^{6-8}$. La gatita, cuya memoria es el objeto de este ejercicio (Figura 1), entró a mi vida a través de la compasión por ella de mi esposa, quien la vio deambular alrededor de nuestra casa por varios años en compañía de otros felinos que vivían libres y salvajes en los jardines y arboledas de nuestro entorno. 


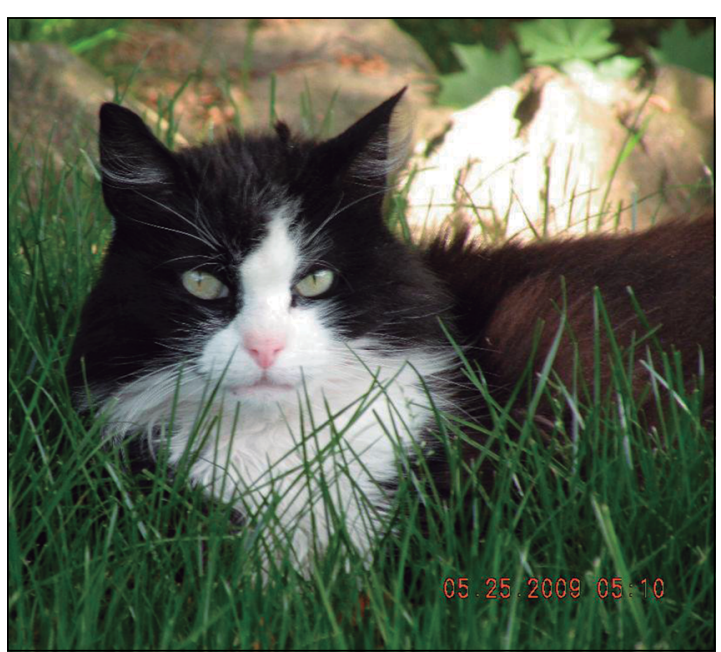

Figura 1. Minicat (Princess).

Esta pequeña colonia de gatos (4-5), de la cual la gatita era un miembro más, estaba constituida por gatos probablemente abandonados por sus dueños y que sobrevivían todo el año a la intemperie. Incluyendo los duros y nevados inviernos neoyorkinos, en una mezcla de vida silvestre y parcial domesticidad y con una habilidad admirable para continuar relativamente imperturbable su riesgosa vida. Este grupo de gatos como otros, estaban protegidos por las leyes de respeto a los animales de Estados Unidos de Norteamérica y por el amparo provisto por los vecinos del lugar, que se preocupaban al igual que nosotros de su alimentación e incluso a veces de su cuidado veterinario comprendiendo esterilizaciones y vacunas; especialmente entre estas últimas la de la rabia, enfermedad que es endémica en los animales silvestres en Nueva York y con los cuales los felinos de vida libre se conectan epidemiológicamente. Estas actividades están consideradas dentro de programas muy populares en Estados Unidos de Norteamérica para proteger a estos animales abandonados, evitando de este modo su cruel eutanasia y la propagación de zoonosis, y que se conocen con el nombre de atrapar-esterilizar-vacunar y libe$\operatorname{rar}^{9,10}$. Sin embargo, a menudo y especialmente en el invierno, algunos de los miembros de la colonia identificados por el color de su pelaje (Blanquito, Negra y Blanca, Naranja) desaparecían, probablemente víctima de las enfermedades propias de la estación o presa de otros predadores mayores como los coyotes, animales también protegidos legalmente que en su búsqueda de comida merodean por los alrededores de las viviendas humanas. Mi esposa, se percató después de algunos años que la gatita estaba envejeciendo, y se preocupó porque ella pudiera pasar otro invierno a la intemperie y para evitar aquello reclutó a voluntarios que trabajaban en la protección de animales para tratar de atraparla, llevarla al veterinario y explorar si se podía alcanzar con ella algún grado mayor de domesticación que pudiera facilitar su introducción a nuestro hogar y prolongar su vida. Después de varios intentos, este objetivo finalmente fue logrado, y el animalito fue llevado al veterinario quien cuidó de su dentadura, recortó su pelaje hirsuto y le administró sus vacunas, además, de hacerle ensayos de laboratorio para descartar la presencia del virus de la leucemia felina que es común en poblaciones de gatos silvestres en los Estados Unidos de Norteamérica.

Posteriormente, mi esposa le construyó una vivienda adecuada en el garaje de nuestra casa para facilitar su domesticación y el animalito comenzó a adaptarse pasando algunas horas del día en ella y saliendo a los alrededores del lugar, especialmente en la noche. Pasado algunos meses se convirtió en un habitante permanente del garaje, con cortas escapadas a las afueras y con una gran preocupación por su alimento y su bienestar, comenzando a dormir largas horas sin sobresaltos, despertándose solo para comer, como si estuviera recuperándose de su larga vida de aventuras en las afueras. Desgraciadamente no la pudimos introducir a la casa, ya que en esa época teníamos un gato muy regalón y territorial que llevaba 14 años viviendo con nosotros y que manifestaba una alta agresividad a cualquier invasor, felino o no felino, de su espacio vital. Pasaron cuatro años, y los dos gatos permanecieron separados, una en el garaje y el otro dentro de casa, hasta que el totalmente domesticado falleció a los 18 años de insuficiencia renal ${ }^{11}$. Sin embargo, a pesar de eso, la gatita prefirió seguir viviendo en sus acomodaciones del garaje explorando la casa solo en la obscuridad solitaria de la noche, viviendo casi cinco años más en esas condiciones de independencia e interactuando físicamente mayoritariamente con mi esposa $\mathrm{y}$ en los dos últimos años antes de su muerte, ocasionalmente conmigo.

Los estudios de paleontología filogenética de 
la evolución de los gatos han establecido que su domesticación fue más difícil que lo que pareciera imaginable ya que ellos revierten fácilmente a la vida silvestre después de esta domesticación ${ }^{6-8}$. Esto explica entonces las colonias de gatos que se tornan silvestres cuando son abandonados por sus amos y de la que fuera un miembro el sujeto de este ensayo ${ }^{6,7}$. Es justamente este ciclo entre la domesticidad y la vida silvestre lo que ha favorecido la infección de los gatos con el gran número de patógenos zoonóticos que pueden portar y los cuales adquieren generalmente a través de sus contactos con animales silvestres ${ }^{1-4}$. Sin embargo, no es menos cierto que la tenencia responsable y civilizada de estos animales y su cuidado diario, y por supuesto su atención veterinaria adecuada y periódica, pueden prevenir muchas de estas infecciones zoonóticas y su potencial contagio a los seres humanos de acuerdo al paradigma de Una Salud ${ }^{5}$. De tal modo que la prevención de las zoonosis trasmitidas por estos animales es una responsabilidad humana y también un deber ético para con estos inteligentes y gráciles seres sentientes que son capaces de aportar tantos contenidos emocionales positivos a nuestras vidas ${ }^{5}$. Enriqueciendo de esta manera nuestra salud afectiva de manera tangible, como sucediera con la inolvidable e inteligente gatita de este relato que respondiera al nombre de Minicat (Princess) (Figura 1). En otro artículo examiné el rol creciente que los gatos están teniendo en la medicina para mejorar el estado psicológico y físico de pacientes con enfermedades tan variadas como la depresión y las dolencias cardiovasculares e incluso para pronosticar el final de la vida, como sucediera con el gato Oscar discutido hace algunos años atrás en el New England Journal of Medicine $e^{11-16}$. Es de esperar que estos positivos programas continúen en aumento, ya que, indudablemente, son beneficiosos para la salud humana y para la de estos animales, ya que permiten descubrir aspectos desconocidos de la biología y de la medicina en ambos grupos.

En el último tiempo, otro problema médico que se ha generado con los gatos y otros animales domésticos es la aparición en ellos de patógenos zoonóticos resistentes a los antimicrobianos, potencialmente trasmisibles a humanos, tales como Salmonella, Escherichia coli y Staphylococcus aureus, entre muchos ${ }^{17,18}$. Sin embargo, aquí nuevamente este inconveniente es un problema de la práctica de la medicina veterinaria y de la humana, que a menudo usan estas drogas maravillosas de manera ilimitada, sin tener en cuenta el problema de la resistencia que afecta negativamente tanto a la salud de los animales como a la de los humanos ${ }^{17,18}$. El animalito conmemorado en este texto fue para mí una gran compañía y una fuente de variadas satisfacciones emocionales; su presencia diaria a la ida y a la vuelta de mi trabajo y en la noche y durante los fines de semana, fuera y dentro de casa, estimulaba mi afectividad y mi imaginación de tal modo que su pasar ha dejado un vacío irreparable. Estas interacciones me imagino eran recíprocas ya que a medida que pasaba el tiempo la docilidad de la pequeña Minicat (Princess) aumentaba, como se desarrollaba también su necesidad y su facilidad para moverse entre humanos. Pero, sin embargo, a pesar de ser un personaje muy querido y regaloneado, nunca en los casi nueve años que vivió con nosotros fue un animal totalmente doméstico y tal vez este aspecto de su vida era parte de su encanto y de su misterio. Esto también demostraba a mi juicio, las interacciones entre los genes de sus antepasados silvestres nacidos en el desierto libio, en África, con el efecto limitado de nuestro ambiente doméstico, que tal vez debido a sus vivencias silvestres previas, fracasó en producir en ella una absoluta domesticación. Esta completa domesticación tal vez habría facilitado la prevención y el diagnóstico de su enfermedad final.

Sin lugar a dudas, que por todas las satisfacciones que estos compañeros nos producen, la tenencia responsable de ellos y sus cuidados veterinarios adecuados dentro del concepto ético y de salud pública de Una Salud ${ }^{5,19,20}$, son lo menos que debemos ofrecerle por la manera fiel y entrañable con que iluminan nuestras vidas y también para el beneficio que nuestras asociaciones con ellos proveen para nuestra salud y bienestar ${ }^{11-17}$. Desearía terminar este breve ensayo con un fragmento del poema de Pablo Neruda "Oda al gato" que capta certera y líricamente carices singulares y seductores de estos queridos animalitos e ilustrándolo con dos pinturas (Figuras 2 y 3 ), de las muchas que hizo de gatos el gran pintor alemán prematuramente fallecido en la batalla de Verdún (1916), durante la Primera Guerra Mundial: Franz Marc, que al igual que Neruda captó con su visión y diestro pincel la gracia, la independencia, la elegancia y el misterio de estos animales ${ }^{20}$. 


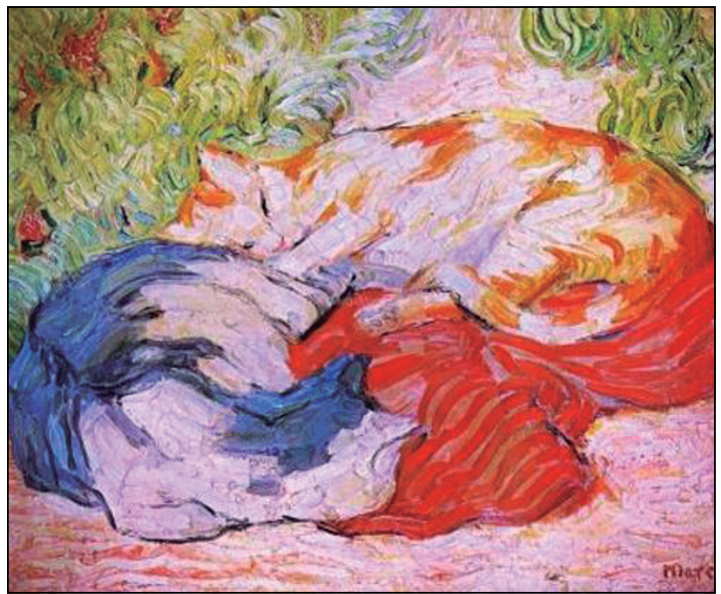

Figure 2. Gatos, Franz Marc (Colección privada).

El hombre quiere ser pescado y pájaro, la serpiente quisiera tener alas, el perro es un león desorientado, el ingeniero quiere ser poeta, la mosca estudia para golondrina, el poeta trata de imitar la mosca, pero el gato quiere ser sólo gato $\mathrm{y}$ todo gato es gato desde bigote a cola, desde presentimiento a rata viva, desde la noche hasta sus ojos de oro.

Agradecimientos: Agradezco a mi esposa Lilo Aron, su preocupación perseverante por los animales y por la feliz introducción de Minicat (Princess) a mi vida. También agradezco a nuestros vecinos sus preocupaciones por la vida y el bienestar de numerosos gatos anónimos, fuentes de innumerables y gratificantes experiencias.

\section{Referencias}

1. Goldstein EJ, Abrahamian FM. Diseases transmitted by cats. Microbiol Spectr 2015; 3 (5). doi: 10.1128/microbiolspec.IOL5-0013-2015. Review. PubMed PMID: 26542039.

2. Chomel BB. Emerging and re-emerging zoonoses of dogs and cats. Animals (Basel) 2014; 4 (3): 434-45. doi:

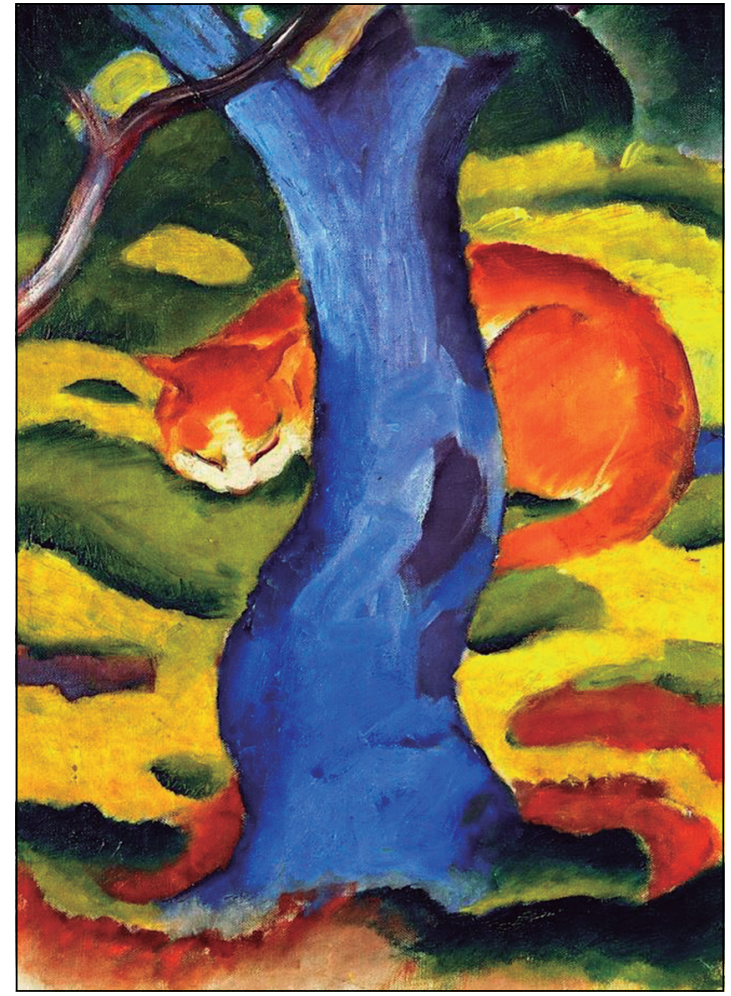

Figura 3. Gato, Franz Marc (Museo Sprengel, Hannover, Alemania).

10.3390/ani4030434. Review. PubMed PMID: 26480316; PubMed Central PMCID: PMC4494318.

3. Gerhold RW, Jessup DA. Zoonotic diseases associated with free-roaming cats. Zoonoses Public Health 2013; 60 (3): 189-95. doi: 10.1111/j.1863-2378.2012.01522.x. Epub 2012 Jul 26. Review. PubMed PMID: 22830565.

4. Damborg P, Broens EM, Chomel BB, Guenther S, Pasmans F, Wagenaar JA, et al. Bacterial zoonoses transmitted by household pets: State-of-the-art and future perspectives for targeted research and policy actions. J Comp Pathol 2016; 155 (1 Suppl 1): S27-40. doi: 10.1016/j.jcpa.2015.03.004. Epub 2015 May 7. PubMed PMID: 25958184.

5. van Herten J, Bovenkerk B, Verweij M. One Health as a moral dilemma: Towards a socially responsible zoonotic disease control. Zoonoses Public Health 2018 Nov 2. doi: 10.1111/zph.12536. [Epub ahead of print] Review. PubMed PMID: 30390380.

6. Ottoni C, van Neer W, De Cupere B, Daligault J, Guimaraes S, Peters J, et al. Of cats and men: the paleogenetic history of the dispersal of cats in the ancient world. bioR ${ }_{\mathrm{X}}$ iv. doi: https://doi.org/10.1101/080028. 
7. Kurushima JD, Ikram S, Knudsen J, Bleiberg E, Grahn RA, Lyons LA. Cats of the Pharaohs: Genetic comparison of Egyptian cat mummies to their feline contemporaries. J Archaeol Sci 2012; 39 (10): 3217-23. Epub 2012 May 19. PubMed PMID: 22923880; PubMed Central PMCID: PMC3426309.

8. Driscoll CA, Macdonald DW, O'Brien SJ. From wild animals to domestic pets, an evolutionary view of domestication. Proc Natl Acad Sci USA 2009; 106 Suppl 1: 9971-8. doi: 10.1073/pnas.0901586106. Epub 2009 Jun 15. PubMed PMID: 19528637; PubMed Central PMCID: PMC2702791.

9. Spehar DD, Wolf PJ. An examination of an iconic trap-neuter-return program: The Newburyport, Massachusetts case study. Animals: an Open Access Journal from MDPI 2017; 7 (11): 81. doi:10.3390/ani7110081.

10. Boone JD. Better trap-neuter-return for free-roaming cats: Using models and monitoring to improve population management. J Feline Med Surg 2015; 17 (9): 8007. doi: 10.1177/1098612X15594995. Review. PubMed PMID: 26323805.

11. Cabello CF. Thoughts on the complex relationship between medicine and animals: a death prayer for a loyal cat. Rev Med Chile 2013; 141 (11): 1449-55. doi: 10.4067/S0034-98872013001100012. Spanish. PubMed PMID: 24718472.

12. Chowdhury EK, Nelson MR, Jennings GL, Wing LM, Reid CM; ANBP2 Management Committee. Pet ownership and survival in the elderly hypertensive population. J Hypertens 2017; 35 (4): 769-75. doi: 10.1097/ HJH.0000000000001214. PubMed PMID: 28009706.

13. Ogechi I, Snook K, Davis BM, Hansen AR, Liu F, Zhang J. Pet ownership and the risk of dying from cardiovascular disease among adults without major chronic medical conditions. High Blood Press Cardiovasc Prev 2016; 23 (3): 245-53. doi: 10.1007/s40292-016-0156-1. Epub 2016 May 12. PubMed PMID: 27174431.
14. Serpell J. Beneficial effects of pet ownership on some aspects of human health and behaviour. J R Soc Med 1991; 84 (12): 717-20. PubMed PMID: 1774745; PubMed Central PMCID: PMC1295517.

15. Litchfield CA, Quinton G, Tindle H, Chiera B, Kikillus $\mathrm{KH}$, Roetman P. The 'Feline Five': An exploration of personality in pet cats (Felis catus). PLoS One 2017; 12 (8): e0183455. doi: 10.1371/journal.pone.0183455. eCollection 2017. PubMed PMID: 28832622; PubMed Central PMCID: PMC5568325.

16. Dosa DM. A day in the life of Oscar the cat. N Engl J Med. 2007; 357 (4): 328-9. PubMed PMID: 17652647.

17. Marques C, Gama LT, Belas A, Bergström K, Beurlet S, Briend-Marchal A, et al. European multicenter study on antimicrobial resistance in bacteria isolated from companion animal urinary tract infections. BMC Vet Res 2016; 12 (1): 213. PubMed PMID: 27658466; PubMed Central PMCID: PMC5034465.

18. Walther B, Tedin K, Lübke-Becker A. Multidrug-resistant opportunistic pathogens challenging veterinary infection control. Vet Microbiol 2017; 200: 71-8. doi: 10.1016/j.vetmic.2016.05.017. Epub 2016 May 27. Review. PubMed PMID: 27291944.

19. Cunningham AA, Daszak P, Wood JLN. One Health, emerging infectious diseases and wildlife: two decades of progress? Philos Trans R Soc Lond B Biol Sci. 2017; 372 (1725). pii: 20160167. doi: 10.1098/rstb.2016.0167. Review. PubMed PMID: 28584175; PubMed Central PMCID: PMC5468692.

20. Destoumieux-Garzón D, Mavingui P, Boetsch G, Boissier J, Darriet F, Duboz P, et al. The One Health Concept: 10 Years Old and a Long Road Ahead. Front Vet Sci 2018; 5: 14. doi: 10.3389/fvets.2018.00014. eCollection 2018. Review. PubMed PMID: 29484301; PubMed Central PMCID: PMC5816263.

21. Partsch S. Franz Marc. Taschen Books. Cologne. Germany. 2001. 